\title{
3-D Model-Based Catheter Tracking for Motion Compensation in EP Procedures
}

\author{
Alexander Brost ${ }^{a}$, Rui Liao ${ }^{b}$, Joachim Hornegger $^{a}$ and Norbert Strobel $^{c}$ \\ ${ }^{a}$ Pattern Recognition Lab, Friedrich-Alexander-University \\ Erlangen-Nuremberg, Erlangen, Germany \\ ${ }^{b}$ Siemens Corporate Research, Princeton, NJ, USA \\ ${ }^{c}$ Siemens AG, Healthcare Sector, Forchheim, Germany
}

\begin{abstract}
Atrial fibrillation is the most common sustained heart arrhythmia and a leading cause of stroke. Its treatment by radio-frequency catheter ablation, performed using fluoroscopic image guidance, is gaining increasingly more importance. Two-dimensional fluoroscopic navigation can take advantage of overlay images derived from pre-operative 3-D data to add anatomical details otherwise not visible under X-ray. Unfortunately, respiratory motion may impair the utility of these static overlay images for catheter navigation. We developed an approach for image-based 3-D motion compensation as a solution to this problem. A bi-plane C-arm system is used to take X-ray images of a special circumferential mapping catheter from two directions. In the first step of the method, a 3-D model of the device is reconstructed. Three-dimensional respiratory motion at the site of ablation is then estimated by tracking the reconstructed catheter model in 3-D. This step involves bi-plane fluoroscopy and 2-D/3-D registration. Phantom data and clinical data were used to assess our model-based catheter tracking method. Experiments involving a moving heart phantom yielded an average 2-D tracking error of $1.4 \mathrm{~mm}$ and an average 3-D tracking error of $1.1 \mathrm{~mm}$. Our evaluation of clinical data sets comprised 469 bi-plane fluoroscopy frames (938 monoplane fluoroscopy frames). We observed an average 2-D tracking error of $1.0 \mathrm{~mm} \pm 0.4 \mathrm{~mm}$ and an average 3-D tracking error of $0.8 \mathrm{~mm} \pm 0.5 \mathrm{~mm}$. These results demonstrate that model-based motion-compensation based on 2-D/3-D registration is both feasible and accurate.
\end{abstract}

Keywords: Motion Compensation, Catheter Tracking, Catheter Model, Atrial Fibrillation

\section{INTRODUCTION}

In 2009, about two million people in the USA are affected by some form of atrial fibrillation (AF). Radio-frequency catheter ablation (RFCA) has become an accepted option for treating AF in today's electrophysiology (EP) labs, in particular, if drug treatment has become ineffective [1, 2]. When catheter ablation of the pulmonary veins (PVs) is carried out under fluoroscopic guidance, image integration combining pre-operative high-resolution 3-D atrial CT and/or MR volumes with the fluoroscopic images can be used (fluoroscopic overlay image guidance) to overcome the problem that X-ray projection images can not distinguish soft tissue well. The potential advantage of this strategy is the fused display of the actual, real-time fluoroscopic images together with images from CT or MRI depicting high soft-tissue contrast [3,4]. Stateof-the art C-arm systems facilitating 3-D tomographic reconstruction can also be used to obtain volumetric data sets of the heart [5-7]. Two examples of bi-plane C-arm systems suitable for fluoroscopic overlay image guidance are shown in Figures 1 and 2 (Artis zee C-arm systems, Siemens AG, Forchheim, Germany). In what follows, the floor-mounted C-arm is denoted as

Send correspondence to Alexander Brost: E-mail: Alexander.Brost@informatik.uni-erlangen.de 


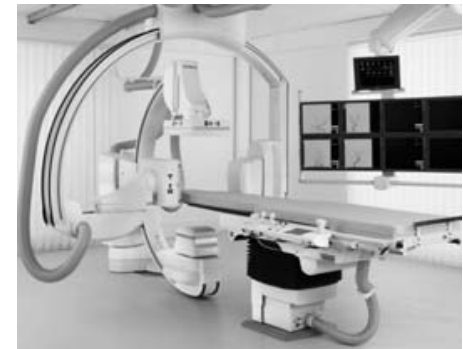

Figure 1: Artis zee Bi-plane with two large flat panel detectors $(30 \times 40 \mathrm{~cm})$.

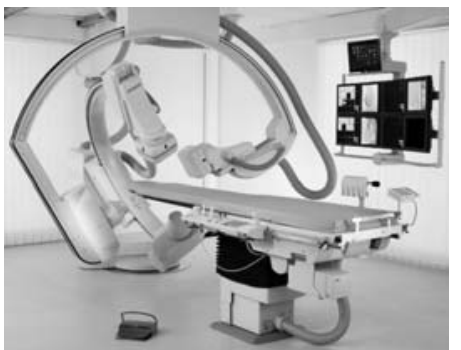

Figure 2: Artis zee Bi-plane with two small flat panel detectors $(20 \times 20 \mathrm{~cm})$.

Plane $A$ and the ceiling-mounted $\mathrm{C}$-arm as Plane $\mathrm{B}$. These descriptions are also used to denote with which $\mathrm{C}$-arm an image or a sequence was taken. When 3-D data is acquired on the Carm device that is also used for 2-D X-ray guidance, registration of the 3-D data set to the 2-D fluoroscopic projections is simplified.

Unfortunately, current fluoroscopic overlay techniques are usually static. As such, they do not account for respiratory and cardiac motion, i.e., they do not follow the heart while it beats and moves through the breathing cycle. Cardiac motion can be taken into account by using electrocardiogram-trigged fluoroscopy acquisition, i.e. synchronizing fluoroscopic images with the electrocardiogram (ECG). In this case, any residual motion is due to breathing, which needs to be compensated to achieve a dynamic fused visualization. Although it has been widely recognized that motion compensation is crucial for fluoroscopic overlays, the first image-based 3-D motion-compensation method for EP applications was just recently proposed in [8], at least to the knowledge of these authors. This may be due to the fact that there are few discernible features in typical EP fluoroscopic images. However, there is literature on dealing with motion for other applications. For example, motion compensated navigation for coronary intervention using magnetic tracking was suggested in [9], but it requires special catheters equipped with an electromagnetic sensor at increased cost. In the papers [10] and [11], vertical motion in the imaging plane was compensated for liver embolization [12] and hepatic artery catheterization, respectively. The first paper involved guidewire tracking, while the second method estimated motion by following the lung-diaphragm interface. In general, though, these methods are not sufficient for EP breathing motion compensation because the heart undergoes a three dimensional motion during respiration $[13,14]$. As a consequence, motion estimation and subsequent compensation in 3-D is indispensable for accurate device navigation near or inside the left atrium (LA) throughout the breathing cycle, e.g. for correct recording of ablation points.

The paper is organized as follows: First, we describe how we generate a 3-D model of the circumferential mapping catheter (CMC) from two views. Then we discuss model-based catheter tracking by 2-D/3-D image registration. Afterwards, we evaluate our method. Finally, we present our results, discuss them, and draw some conclusions.

\section{METHODS}

\subsection{Circumferential Mapping Catheter Model Generation in 3-D}

Our model generation of the circumferential mapping catheter (CMC) is based on the following assumptions:

1. The CMC is approximated as circular when positioned at the ostium of the PV.

2. The $\mathrm{CMC}$ can be approximated by an ellipse in 3-D.

3. The projections of the CMC into 2-D (X-Ray) images are approximated as 2-D ellipses. 


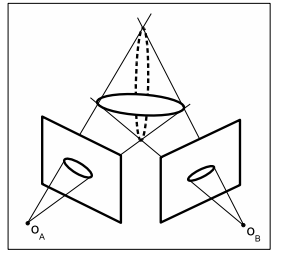

(a)

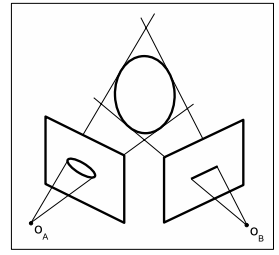

(b)

Figure 3: 3-D mapping catheter model initialization from two views. Figure 3(a): This general case shows two possible (dual) solutions when reconstructing a 3-D ellipse from bi-plane 2-D ellipses. The correct solution can be found by using prior knowledge, e.g., of the diameter of the circumferential mapping catheter. Figure 3(b): This special case reconstructs a 3-D ellipse from one 2-D ellipse in one X-ray view and a line in the other.

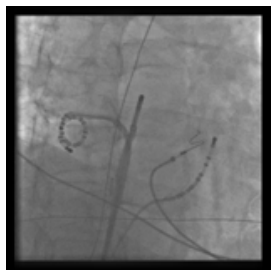

(a) Plane A

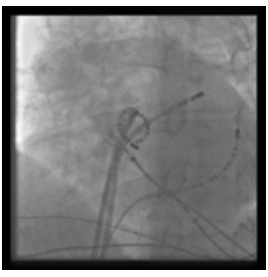

(b) Plane B

Figure 4: General case: the bi-plane C-arm system is set up such that the circular mapping catheter is projected as an ellipse in each view. This situation can occur if the bi-plane view directions are not adjusted to a patient's anatomy (left atrium, pulmonary veins). Since a catheter has to be moved through the vessels of the body to reach its target, a circular catheter has to have a linear shape including a catheter tip.

For the last assumption, we also accept the special case of a 2-D line as an ellipse with one half-axis being 0 . The case arises under some special $\mathrm{C}$-arm viewing directions, as shown in Figure 5. For the model generation, we differentiate between the regular case and the special case as shown in Figure 3. Note that we do not consider the case that the 3-D mapping catheter becomes a line in both X-ray projections as this is a very undesirable case in a clinical environment and can be omitted during clinical procedures, by adjusting one of the $\mathrm{C}$-arms.

For the reconstruction it is essential to know the 2-D ellipse parameters. To get the projection of the circumferential mapping catheter on the imaging plane, the catheter is first extracted by manual clicking followed by fast marching in one frame of the fluoroscopy sequence, as explained in [15]. The 2-D ellipses are then calculated such that all ellipse points satisfy the linear equation [16]

$$
a u_{i}^{2}+b u_{i} v_{i}+c v_{i}^{2}+d u_{i}+e v_{i}+f=0
$$

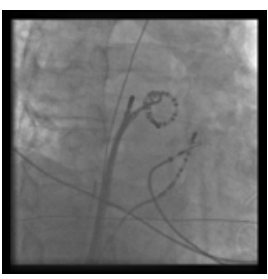

(a) Plane A

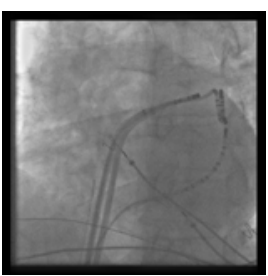

(b) Plane B

Figure 5: Special case: this viewing configuration can simplify bi-plane catheter navigation during ablation, where the circular mapping catheter often serves as a visual reference. In this view setup, the physician needs to verify that the ablation catheter is in the vicinity of the CMC's elliptical projection in one view and close to the line in the other. 
with the 2-D coordinates of the ellipse $\left(u_{i}, v_{i}\right)$ and the coefficients of the ellipse $a, b, c, d, e, f \in$ $\mathbb{R}$, usually combined with the constraint $\|\mathbf{f}\|_{2}=1$. As Eq. (1) does not only describe ellipses, but also hyperbolas and parabolas, we need to incorporate another constraint to ensure that our solution is elliptical.The method presented in [16] assures an elliptical solution by enforcing the condition $b^{2}-4 a c<0[17,18]$. Eq. (1) can be rewritten as

$$
\left(\begin{array}{lll}
u_{i} & v_{i} & 1
\end{array}\right) \cdot \mathbf{C} \cdot\left(\begin{array}{lll}
u_{i} & v_{i} & 1
\end{array}\right)^{T}=0
$$

with $\mathbf{C} \in \mathbb{R}^{3 \times 3}$ representing the implicit ellipse parameters. When fitting a regular ellipse, the rank of $\mathbf{C}$ is 3 . For the special case, when a line is fitted to satisfy Eq. (1), the resulting matrix $\mathrm{C}$ is rank-deficient.

As the ellipse points are projections of a circular shaped object in 3-D space, we can write

$$
\left(\begin{array}{llll}
x_{i} & y_{i} & z_{i} & 1
\end{array}\right) \cdot \mathbf{P}^{T} \cdot \mathbf{C} \cdot \mathbf{P} \cdot\left(\begin{array}{llll}
x_{i} & y_{i} & z_{i} & 1
\end{array}\right)^{T}=0
$$

with the projection matrix $\mathbf{P} \in \mathbb{R}^{3 \times 4}$ having rank of 3 . The matrix product $\mathbf{P}^{T} \cdot \mathbf{C} \cdot \mathbf{P}$ is replaced by $\mathbf{Q} \in \mathbb{R}^{4 \times 4}$, describing a cone in 3-D space with the optical center as origin and passing through the ellipse in the imaging plane. Given two projection images of an ellipse in 3-D on both A-plane view and B-plane view of a bi-plane system, respectively, we get two 3-D cones denoted $\mathbf{Q}_{A}$ for imaging plane $A$ and $\mathbf{Q}_{B}$ for imaging plane $B$. The representations of $\mathbf{Q}_{A}$ and $\mathbf{Q}_{B}$ are quadrics of rank 3. The reconstructed ellipse is then computed by calculating a $\lambda$ such that the quadric statisfies [19]

$$
\operatorname{rank}\left(\mathbf{Q}_{A}+\lambda \cdot \mathbf{Q}_{B}\right)=2
$$

A quadric of rank 2 describes two intersecting planes, which are extracted by an eigenvalue and eigenvector analysis. Each of these planes contains a valid ellipse in 3-D that projects onto the ellipses in the imaging planes, see Figure 3. As we require only one solution for our tracking approach, we utilize our prior knowledge about the anatomy of the pulmonary veins and select the result that is more circular, because the $\mathrm{CMC}$ inserted into a PV resembles a circle more closely than an ellipse in normal human anatomy. The circularity is determined by:

$$
\kappa=|\phi-\psi|
$$

with the axes $\phi$ and $\psi$ of an ellipse. To obtain the more circular solution, the ellipse with the smaller value for $\kappa$ is used. For the special case where the circumferential mapping catheter is projected close to being a line in one view, the method in [19] is not stable, as this method requires for $\mathbf{Q}_{A}$ and $\mathbf{Q}_{B}$ to be of rank 3. Unfortunately, if a line is described by the matrix $\mathbf{C}$, its rank is reduced to 2 . Thus the multiplication of $\mathbf{P}^{T} \cdot \mathbf{C} \cdot \mathbf{P}$ leads to a matrix of rank 2. This special case is detected during ellipse fitting. In this case, we propose to reconstruct the 3-D mapping catheter model as follows. First, a line is fitted to the line-like projection of the mapping catheter. It is calculated as the the principal axis of the points obtained by fast marching. Two arbitrary but distinct points $\mathbf{q}_{1}$ and $\mathbf{q}_{2}$ are then randomly selected on the fitted line and are connected to the optical center. The projection plane in which both the X-ray source and the fitted line lies is then determined by the two rays $\mathbf{q}_{1, o}$ and $\mathbf{q}_{2, o}$. In the second and final step, the 3-D mapping catheter model is obtained by intersecting this plane with the elliptical cone defined by the ellipse from the second view.

\subsection{Model-Based Catheter Tracking}

After the 3-D model of the circumferential mapping catheter has been generated from the first frame of the fluoroscopic sequence, it is tracked in 3-D throughout the remainder of the biplane sequence by performing 2-D/3-D registration on pre-processed X-ray images. The use of 


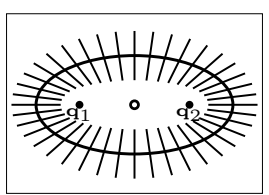

Figure 6: Two-dimensional ellipse with center (circle), focal points (dots), $\mathbf{q}_{1}$ and $\mathbf{q}_{2}$, and normals.

a bi-plane system facilitates the estimation of a 3-D motion, but techniques are conceivable to perform motion-compensation from one direction as well. In either case, it is essential to establish benchmark results using bi-plane imaging. The term images refers to the corresponding frames of viewing plane $A$ and of viewing plane $B$, respectively. The images are pre-processed to improve the quality of the registration. In the first pre-processing step, the region of interest (ROI) for tracking is cut to $400 \times 400$ pixels (on the $1024 \times 1024$ image) around the center of the tracked mapping catheter in the previous frame. This speeds up the algorithm, and it also minimizes the influence of peripheral structures that could interference with catheter tracking. Histogram equalization on the ROI is applied next to increase image contrast. In addition, a vessel enhancement filter is used to enhance line-like structures such as the circumferential mapping catheter [20]. The feature image is then binarized using Otsu's algorithm [21]. This facilitates segmentation of the mapping catheter. Finally, a distance map is calculated from the binarized image [22]. The distance map encodes the distance from a point to its closest feature point, that is the nonzero point representing the extracted mapping catheter in our binarized feature image. The distance transform offers an important advantage. It provides a denoised representation of the fluoroscopic image with a pronounced minimum around the 2-D shape of the circumferential mapping catheter. This is why, we can still reach a good registration, even if the 3-D model is not $100 \%$ accurate, or if we start from a position that is somewhat distant from the mapping catheter to be tracked.

Model-based catheter tracking in 3-D is achieved by performing 2-D/3-D registration. To this end, the reconstructed 3-D catheter model is rotated by $\mathbf{R} \in \mathbb{R}^{4 \times 4}$ and translated by $\mathbf{T} \in$ $\mathbb{R}^{4 \times 4}$ in 3-D first. Then it is projected onto the two imaging planes of the bi-plane C-arm system. The average distance between the projected points and the closest feature point (i.e. the circumferential mapping catheter) in fluoroscopic images is efficiently calculated using the distance map introduced above. A suitable rotation and translation is found by optimizing

$$
\hat{\mathbf{R}}, \hat{\mathbf{T}}=\arg \min _{\mathbf{R}, \mathbf{T}} \sum_{i} \mathbf{I}_{\mathrm{DT}}\left(\mathbf{P}_{A} \cdot \mathbf{T} \cdot \mathbf{R} \cdot \mathbf{w}_{i}\right)+\sum_{i} \mathbf{I}_{\mathrm{DT}}\left(\mathbf{P}_{B} \cdot \mathbf{T} \cdot \mathbf{R} \cdot \mathbf{w}_{i}\right) .
$$

The parameters used for optimizing are three rotation angles around the main axes in 3-D, combined in one rotation matrix $\mathbf{R}$, as well as a three-dimensional translation, represented as matrix T. However, due to the fact that the shape of mapping catheters may not always be exactly elliptical, a simple elliptical 3-D model may not fit perfectly. Fortunately, the smoothness of the distance transform helps to find an optimum close to the exact position, facilitating a robust tracking. To improve 2-D/3-D registration, the diameter of the circumferential mapping catheter is taken into account as well. It ranges from $4 \mathrm{~F}$ to $7 \mathrm{~F}^{*}$. This information is used as a constraint along the normals of the ellipses, as shown in Fig. 6. This information about the diameter of the catheter could be obtained, e.g. by user input, or a conservative estimate could be used, e.g. 7 F. Due to the smoothness of the distance map, the addition of more pixels improves the registration quality, as the semi-automatic segmentation does not result in a single line, but a rather broad line that is then used for the distance transform.

\footnotetext{
${ }^{*}$ The French catheter scale is defined as $\frac{1}{3}[\mathrm{~mm}]=1 \mathrm{Fr}$.
} 


\begin{tabular}{|l|c|c|c|c|}
\hline \multicolumn{5}{|c|}{ Simulation - Model Generation Error } \\
\hline & No Noise & Noise & Offset & Noise and Offset \\
\hline General Case & $0.2 \mathrm{~mm}$ & $2.8 \mathrm{~mm}$ & $5.0 \mathrm{~mm}$ & $5.3 \mathrm{~mm}$ \\
\hline Special Case & $0.3 \mathrm{~mm}$ & $0.4 \mathrm{~mm}$ & $2.1 \mathrm{~mm}$ & $2.1 \mathrm{~mm}$ \\
\hline
\end{tabular}

Table 1: Simulation results for 3-D catheter model generation from bi-plane views. The errors listed in the table were calculated by averaging individual errors over five circles reconstructed from all angulations considered. Gaussian noise with zero-mean and a standard deviation of $2.0 \mathrm{~mm}$ was used to disturb the points. The offset was set at $2.0 \mathrm{~mm}$ as well.

\section{EXPERIMENTS AND RESULTS}

\subsection{Model Generation}

Our goal is to estimate motion by registering a 3-D model of a circular mapping catheter to two associated 2-D projections taken simultaneously under two different viewing angles. Biplane X-ray imaging systems are used as displayed in Figures 1 and 2. Rigid three-dimensional motion can be estimated, by successively estimating the 3-D position of the center of the ellipse after successful registration. This approach depends on an accurate estimate for a model of the circumferential mapping catheter used. In what follows, we evaluate the accuracy of our catheter model generation step. We start with simulations before we turn to experiments involving actual X-ray projections. In our simulations, five circles in 3-D space were set up, each with a different position, orientation and diameter. These five 3-D circles were forward projected onto 2-D imaging planes for a set of $\mathrm{C}$-arm projection angles. In the next step, these 3-D circles were reconstructed by triangulation. The reconstruction was done for the $\mathrm{C}$-arm position angles (RAO/LAO), (CRAN/CAUD) $\in\left[-90^{\circ},-60^{\circ},-30^{\circ}, \ldots, 60^{\circ}, 90^{\circ}\right]$. LAO/RAO refers to a left/right anterior oblique detector position, and CRAN/CAUD means cranial/caudal or head/feet viewing orientation with respect to the patient. The space of all possible $\mathrm{C}$-arm detector positions was subsampled in steps of $30^{\circ}$, given a minimum angular difference of $30^{\circ}$ and a maximum difference of $150^{\circ}$ between two $\mathrm{C}$-arm views used for 3-D ellipse reconstruction by triangulation. The cases considering an angular difference of $0^{\circ}$ and $180^{\circ}$, respectively, were omitted during the simulation. Not all of these viewing angles are useful in a clinical environment, but the results give a systematic evaluation of the accuracy of our reconstruction method. The overall error is calculated as the average distance between points on the original 3-D circle and their nearest-neighbor counterparts on the reconstructed circle. Four cases were evaluated. In the first case, we simply reconstructed a 3-D circle from the projection images not adding any noise to find out how the reconstruction method works in an idealized scenario. In the second case, we added Gaussian noise with zero mean and a standard deviation of $2.0 \mathrm{~mm}$ to the 2-D points before reconstructing the 3-D object. In the third case, we added a translational error in one imaging plane of $2.0 \mathrm{~mm}$. The fourth case involved both Gaussian noise and translational error. The results are summarized in Table 1 . The errors listed in the table were calculated by averaging individual errors over five circles reconstructed from all bi-plane C-arm angulations considered. The general case refers to the situation where an ellipse was visible in both imaging planes. The special case implies that there was one ellipse in one view, while it collapsed to a line in the other view. The projection matrices for the simulation were computed as described in $[23,24]$.

Our simulation results show that 3-D reconstruction is very accurate under ideal conditions, but deteriorates noticeably when there is noise and 3-D reconstruction based on in the general view configuration. If 3-D reconstruction is, however, based on a 3-D ellipse in one view and a line in the other, then 3-D reconstruction remains accurate as displayed in Table 1. From this we conclude that 3-D ellipse reconstruction from two 2-D ellipses is very sensitive to noise in the 2-D points of the detected ellipse. To deal with this problem, high-precision ellipse detection is required for initial model generation in the general case. As an alternative, the bi-plane $\mathrm{C}$-arm 


\begin{tabular}{|c|c|c|c|c|c|}
\hline \multicolumn{7}{|c|}{ Phantom Experiment - Model Generation Error } \\
\hline \multirow{2}{*}{ No. } & \multicolumn{2}{|c|}{ Plane A } & \multicolumn{2}{c|}{ Plane B } & \multirow{2}{*}{ 3-D Error } \\
\cline { 2 - 5 } & Position & 2-D Error & Position & 2-D Error & \\
\hline 1 & $(53 ; 0)$ & $1.3 \mathrm{~mm}$ & $(-36 ; 0)$ & $0.8 \mathrm{~mm}$ & $1.4 \mathrm{~mm}$ \\
\hline 2 & $(-30 ; 0)$ & $0.9 \mathrm{~mm}$ & $(-120 ; 0)$ & $1.1 \mathrm{~mm}$ & $1.7 \mathrm{~mm}$ \\
\hline 3 & $(-42 ; 1)$ & $0.9 \mathrm{~mm}$ & $(-120 ;-1)$ & $1.2 \mathrm{~mm}$ & $1.5 \mathrm{~mm}$ \\
\hline 4 & $(-37 ; 1)$ & $0.9 \mathrm{~mm}$ & $(-120 ;-1)$ & $1.1 \mathrm{~mm}$ & $1.4 \mathrm{~mm}$ \\
\hline 5 & $(-34 ; 1)$ & $0.9 \mathrm{~mm}$ & $(-120 ;-1)$ & $1.2 \mathrm{~mm}$ & $1.7 \mathrm{~mm}$ \\
\hline ave. & - & $\mathbf{1 . 0} \mathbf{~} \mathbf{m m}$ & - & $\mathbf{1 . 1} \mathbf{~} \mathbf{m}$ & $\mathbf{1 . 5} \mathbf{~ m m}$ \\
\hline
\end{tabular}

Table 2: Experimental results for 3-D model generation by triangulation from two views. The given 2-D error represents the mean deviation of the projected 3-D model into each imaging plane from the original 2-D segmentation. We use ave. to denote the average error over the experiments performed.

\begin{tabular}{|c|c|c|c|c|c|c|}
\hline \multicolumn{7}{|c|}{ Phantom Experiment - 2-D Error } \\
\hline \multirow{2}{*}{ No. } & \multicolumn{3}{|c|}{ Plane A } & \multicolumn{3}{c|}{ Plane B } \\
\cline { 2 - 7 } & Mean & Std. & Model Error & Mean & Std. & Model Error \\
\hline 1 & $1.4 \mathrm{~mm}$ & $0.7 \mathrm{~mm}$ & $0.4 \mathrm{~mm}$ & $2.5 \mathrm{~mm}$ & $1.0 \mathrm{~mm}$ & $1.0 \mathrm{~mm}$ \\
\hline 2 & $1.5 \mathrm{~mm}$ & $0.2 \mathrm{~mm}$ & $1.0 \mathrm{~mm}$ & $1.6 \mathrm{~mm}$ & $0.2 \mathrm{~mm}$ & $1.2 \mathrm{~mm}$ \\
\hline 3 & $1.7 \mathrm{~mm}$ & $0.4 \mathrm{~mm}$ & $1.2 \mathrm{~mm}$ & $1.2 \mathrm{~mm}$ & $0.3 \mathrm{~mm}$ & $0.9 \mathrm{~mm}$ \\
\hline 4 & $1.9 \mathrm{~mm}$ & $0.5 \mathrm{~mm}$ & $1.3 \mathrm{~mm}$ & $1.1 \mathrm{~mm}$ & $0.2 \mathrm{~mm}$ & $1.0 \mathrm{~mm}$ \\
\hline 5 & $2.3 \mathrm{~mm}$ & $0.6 \mathrm{~mm}$ & $1.3 \mathrm{~mm}$ & $1.0 \mathrm{~mm}$ & $0.2 \mathrm{~mm}$ & $0.8 \mathrm{~mm}$ \\
\hline ave. & $\mathbf{1 . 6} \mathbf{~} \mathbf{m}$ & $\mathbf{0 . 6} \mathbf{~} \mathbf{m}$ & $\mathbf{1 . 1} \mathbf{~} \mathbf{m}$ & $\mathbf{1 . 2} \mathbf{~} \mathbf{m}$ & $\mathbf{0 . 5} \mathbf{~} \mathbf{m}$ & $\mathbf{1 . 0} \mathbf{~} \mathbf{m}$ \\
\hline
\end{tabular}

Table 3: Average 2-D tracking error for the phantom sequences used. The last row shows an average over all the five sequences for plane A and plane B, respectively. The total number of frames was 173 .

system may be set up such that we have an ellipse in one view and line in the other. In this case, 3-D reconstruction involves a plane intersecting a cone. Much more robust results are obtainable then, as shown in Table 1. If there is an uncompensated translation between the Aplane and B-plane of the bi-plane system, e.g., $2 \mathrm{~mm}$ as used for our experiment, then the 3-D reconstruction error may increase by around the same amount. This implies that the relative lateral position of the A-plane to the B-plane must be known with high precision.

To further validate our approach, we acquired bi-plane fluoroscopic images of a static CMC from different viewing directions and compared the 3-D reconstruction results to a 3-D data set reconstructed using $\mathrm{C}$-arm $\mathrm{CT}$ on the same system. C-arm CT involved X-ray data acquisition on an AXIOM Artis dBA bi-plane system (Siemens AG, Forchheim, Germany). To this end, the A-plane performed a partial circle scan around the experimental setup first. Then, a 3$\mathrm{D}$ data set was reconstructed on a syngo X-Workplace running syngo DynaCT (both Siemens AG, Forchheim, Germany). The 3-D coordinates of the circumferential mapping catheter were obtained from the 3-D volume and compared to the 3-D reconstruction results obtained from biplane views. To mimic a clinical setup, we varied only the primary angle (LAO/RAO), as it would be during an EP procedure. The secondary angle (CRAN/CAU) was kept constant. The experimental results for catheter model generation are presented in Table 2. It can be seen that our 3-D reconstruction algorithm is not sensitive to C-Arm setup and works relatively accurately for all five angulations tested.

\subsection{Model-Based Catheter Tracking}

After a 3-D model of the circumferential mapping catheter has been set up from the first frame of the bi-plane fluoroscopic sequence, the position of this 3-D model is continuously adjusted by performing 2-D/3-D registration. We evaluated our algorithm on five bi-plane phantom fluoroscopy sequences that were acquired using a moving heart phantom to which the mapping catheter was fixed. This was to mimick the situation when the catheter is placed at the ostium 


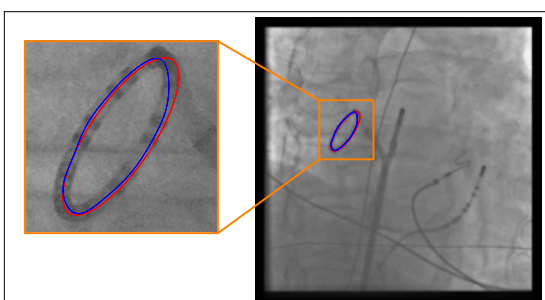

Figure 7: Tracked ellipse in plane A. It has an average distance to the manual segmentation of $1.0 \mathrm{~mm}$ (model error $0.5 \mathrm{~mm}$ ).

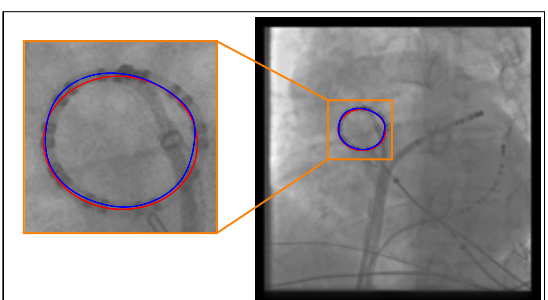

Figure 8: In this particular frame, the tracking error $(0.6 \mathrm{~mm})$ is completely due to the model error $(0.6 \mathrm{~mm})$.

of one of the pulmonary veins. We also calculated the tracking error throughout 13 different clinical fluoroscopic sequences that were acquired during EP procedures on an AXIOM Artis dBA C-arm system (Siemens AG, Forchheim, Germany). We focused on scenes recorded during atrial fibrillation ablation that show one circumferential mapping catheter and one ablation catheter. The presence of other structures did not decrease the accuracy of our method, because we used a unique elliptical structure for registration. To study the tracking error in 2-D, we forward projected the 3-D catheter model, computed from the first frame, into both planes of the bi-plane imaging system after 2-D/3-D registration. The first frame is used to set up a 3-D model of the circumferential ablation catheter. As no 2-D/3-D registration is performed in this particular frame, it is only affected by a model error. The model error expresses how well the 3-D model fits to its associated 2-D projections it was generated from. Starting with the second frame, we calculated the average 2-D distance of the forward projected 3-D catheter model to a manually segmented mapping catheter. An example for a bi-plane frame is presented in Figures 7 and 8. The manual catheter segmentation in each fluoroscopic frame was supervised by a cardiologist, and we consider it our reference result. The 2-D distances between the forward projected 3-D model and the manually segmented reference in each fluoroscopic frame of a sequence were averaged over all frames to arrive at an overall 2-D tracking error for each sequence. It is expressed in terms of mean and standard deviation. To evaluate the 3-D accuracy of our motion estimation approach based on model-based catheter tracking, we selected the tip of the circumferential mapping catheter as a reference point. The tip of a circular catheter can, e.g., be seen nicely in Figure 4. The tip was manually localized throughout all sequences by triangulating its 3-D position from bi-plane frames to get a reference first. In the next step, we applied the motion estimated by catheter tracking to the catheter tip to move it from its 3-D position in the previous frame to the next frame. Finally, we compared the 3-D position reached by applying the estimated motion to the actual 3-D reference point obtained by triangulation $[23,24]$. In the end, the error was calculated as the Euclidean distance in 3-D space.

The 2-D and 3-D tracking error for the circular catheter attached to the moving heart phantom are listed in Table 3 and Table 4, respectively. In Table 3, the 2-D error was computed for each of the two imaging planes, i.e., for Plane A as well as for Plane B. The average over the mean tracking errors obtained for the sequences recorded with the moving heart phantom was $1.6 \mathrm{~mm} \pm 0.6 \mathrm{~mm}$ for plane A and $1.2 \mathrm{~mm} \pm 0.5 \mathrm{~mm}$ for plane B. The A-plane model errors were between $0.4 \mathrm{~mm}$ and $1.3 \mathrm{~mm}$, while the B-plane model errors ranged between $0.6 \mathrm{~mm}$ to

\begin{tabular}{|c|c|c|c|c|c|c|}
\hline \multicolumn{7}{|c|}{ Phantom Experiment - 3-D Error } \\
\hline Seq. No. & 1 & 2 & 3 & 4 & 5 & ave. \\
\hline 3-D Error in [mm] & 1.1 & 1.1 & 1.9 & 0.6 & 1.3 & $\mathbf{1 . 1}$ \\
\hline
\end{tabular}

Table 4: The mean 3-D tracking error in $\mathrm{mm}$ for each phantom sequence, over 173 bi-plane fluoroscopy frames. 


\begin{tabular}{|c|c|c|c|c|c|c|}
\hline \multicolumn{7}{|c|}{ Clinical Data - 2-D Error } \\
\hline \multirow{2}{*}{ No. } & \multicolumn{3}{|c|}{ Plane A } & \multicolumn{3}{c|}{ Plane B } \\
\cline { 2 - 7 } & Mean & Std. & Model Error & Mean & Std. & Model Error \\
\hline 1 & $1.0 \mathrm{~mm}$ & $0.3 \mathrm{~mm}$ & $0.8 \mathrm{~mm}$ & $1.0 \mathrm{~mm}$ & $0.4 \mathrm{~mm}$ & $0.5 \mathrm{~mm}$ \\
\hline 2 & $1.1 \mathrm{~mm}$ & $0.3 \mathrm{~mm}$ & $0.5 \mathrm{~mm}$ & $0.7 \mathrm{~mm}$ & $0.2 \mathrm{~mm}$ & $0.6 \mathrm{~mm}$ \\
\hline 3 & $0.9 \mathrm{~mm}$ & $0.3 \mathrm{~mm}$ & $0.4 \mathrm{~mm}$ & $0.9 \mathrm{~mm}$ & $0.4 \mathrm{~mm}$ & $0.3 \mathrm{~mm}$ \\
\hline 4 & $1.0 \mathrm{~mm}$ & $0.2 \mathrm{~mm}$ & $0.6 \mathrm{~mm}$ & $1.8 \mathrm{~mm}$ & $0.4 \mathrm{~mm}$ & $1.1 \mathrm{~mm}$ \\
\hline 5 & $1.1 \mathrm{~mm}$ & $0.2 \mathrm{~mm}$ & $0.8 \mathrm{~mm}$ & $2.1 \mathrm{~mm}$ & $1.0 \mathrm{~mm}$ & $0.7 \mathrm{~mm}$ \\
\hline 6 & $1.0 \mathrm{~mm}$ & $0.6 \mathrm{~mm}$ & $0.5 \mathrm{~mm}$ & $0.8 \mathrm{~mm}$ & $0.2 \mathrm{~mm}$ & $0.6 \mathrm{~mm}$ \\
\hline 7 & $1.0 \mathrm{~mm}$ & $0.2 \mathrm{~mm}$ & $0.5 \mathrm{~mm}$ & $1.1 \mathrm{~mm}$ & $0.2 \mathrm{~mm}$ & $0.7 \mathrm{~mm}$ \\
\hline 8 & $0.8 \mathrm{~mm}$ & $0.1 \mathrm{~mm}$ & $0.7 \mathrm{~mm}$ & $1.1 \mathrm{~mm}$ & $0.1 \mathrm{~mm}$ & $0.6 \mathrm{~mm}$ \\
\hline 9 & $1.0 \mathrm{~mm}$ & $0.1 \mathrm{~mm}$ & $0.8 \mathrm{~mm}$ & $0.7 \mathrm{~mm}$ & $0.1 \mathrm{~mm}$ & $0.9 \mathrm{~mm}$ \\
\hline 10 & $0.8 \mathrm{~mm}$ & $0.1 \mathrm{~mm}$ & $0.6 \mathrm{~mm}$ & $1.1 \mathrm{~mm}$ & $0.2 \mathrm{~mm}$ & $0.6 \mathrm{~mm}$ \\
\hline 11 & $1.6 \mathrm{~mm}$ & $0.4 \mathrm{~mm}$ & $0.8 \mathrm{~mm}$ & $0.7 \mathrm{~mm}$ & $0.1 \mathrm{~mm}$ & $0.6 \mathrm{~mm}$ \\
\hline 12 & $0.7 \mathrm{~mm}$ & $0.2 \mathrm{~mm}$ & $0.6 \mathrm{~mm}$ & $1.0 \mathrm{~mm}$ & $0.1 \mathrm{~mm}$ & $0.8 \mathrm{~mm}$ \\
\hline 13 & $0.7 \mathrm{~mm}$ & $0.1 \mathrm{~mm}$ & $0.8 \mathrm{~mm}$ & $0.9 \mathrm{~mm}$ & $0.1 \mathrm{~mm}$ & $0.8 \mathrm{~mm}$ \\
\hline ave. & $\mathbf{1 . 0} \mathbf{~ m m}$ & $\mathbf{0 . 4} \mathbf{~ m m}$ & $\mathbf{0 . 6} \mathbf{~ m m}$ & $\mathbf{1 . 0} \mathbf{~ m m}$ & $\mathbf{0 . 4} \mathbf{~ m m}$ & $\mathbf{0 . 7} \mathbf{~ m m}$ \\
\hline
\end{tabular}

Table 5: Average 2-D tracking error for the clinical sequences used. The last row shows an average over all the 13 sequences for plane A and plane B, respectively. The total number of frames was 469 for each imaging plane.

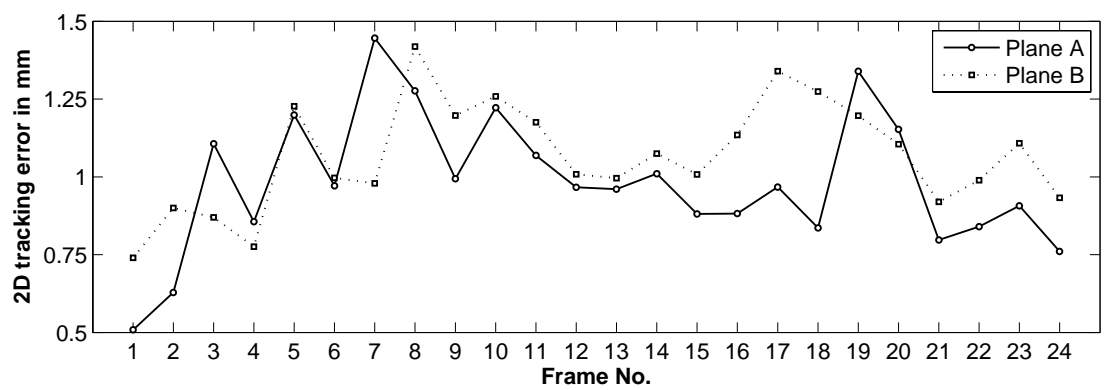

Figure 9: Two-dimensional tracking error in $\mathrm{mm}$ for one sequence (No. 7) frame by frame. The A-plane and B-plane model errors are $0.5 \mathrm{~mm}$ and $0.7 \mathrm{~mm}$, respectively.

\section{$2.1 \mathrm{~mm}$}

Both A-plane and B-plane 2-D tracking results for each of the 13 clinical sequences are summarized in Table 5. The 3-D errors are shown in Table 6. The average over the mean tracking errors obtained for the clinical sequences was $1.0 \mathrm{~mm} \pm 0.4 \mathrm{~mm}$ for plane A and $1.0 \mathrm{~mm} \pm 0.4 \mathrm{~mm}$ for plane B. An example for one frame of one sequence is shown in Figures 7 and 8 . The A-plane model errors were between $0.4 \mathrm{~mm}$ and $0.8 \mathrm{~mm}$, while the B-plane model errors ranged between $0.3 \mathrm{~mm}$ to $1.1 \mathrm{~mm}$. A detailed frame-by-frame 2-D tracking error for sequence no. 7 is presented in Figure 9. The 2-D tracking error over all 469 bi-plane fluoroscopy frames (938 monoplane fluoroscopy frames) was $1.0 \mathrm{~mm} \pm 0.4 \mathrm{~mm}$ (mean \pm std.). Looking at the frame-by-frame tracking error, as shown in Figure 9, we can see that our tracking algorithm

\begin{tabular}{|c|c|c|c|c|c|c|c|}
\hline \multicolumn{1}{|c|}{ Clinical Data - 3-D Error } \\
\hline Seq. No. & 1 & 2 & 3 & 4 & 5 & 6 & 7 \\
\hline 3-D Error in $[\mathrm{mm}]$ & 1.6 & 1.4 & 1.4 & 0.7 & 2.0 & 0.7 & 0.8 \\
\hline Seq. No. & 8 & 9 & 10 & 11 & 12 & 13 & ave. \\
\hline 3-D Error in [mm] & 1.2 & 0.8 & 0.7 & 1.2 & 1.0 & 0.7 & $\mathbf{0 . 8}$ \\
\hline
\end{tabular}

Table 6: The mean 3-D tracking error in $\mathrm{mm}$ for each sequence. 


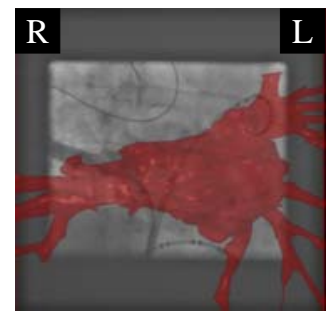

Figure 10: Frame of a non-motion compensated sequence with static fluoroscopic overlay. Note the mismatch between mapping catheter and LA.

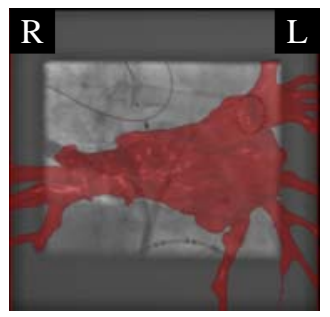

Figure 11: The same sequence with motion compensation. Note how well both mapping catheter and contrast agent are matched to the morphology of the LA.

does not suffer from error propagation. This is due to the tracking by registration approach. Every new frame is processed without incorporating too much knowledge of the previous frame or previous movement. Only the information about the catheter position within the previous frames is used as center of the region of interest in the current frame.

The 3-D error, presented in Table 6, is slightly lower than the 2-D error, presented in Table 5. The reason for this difference is the projection geometry. It magnifies 3-D errors. Note, however, that the error vector in 3-D need not necessarily be parallel to the imaging plane. In this case, the 2-D error associated with the forward projected 3-D error is smaller.

\section{DISCUSSION AND CONCLUSIONS}

We developed a method for 3-D motion estimation for radio-frequency catheter ablation of atrial fibrillation. A bi-plane $\mathrm{X}$-ray $\mathrm{C}$-arm system is used to simultaneously image a circumferential mapping catheter from two directions. Catheter tracking is based on 2-D/3-D registration principles. To this end, a 3-D catheter model is computed from the first frame of a fluoroscopic sequence. Our experiments showed an average Euclidean distance from a real catheter to its reconstructed model of about $1.5 \mathrm{~mm}$. The catheter model is then forward projected onto both imaging planes. There, it is registered by minimizing an error based on a distance map derived from the fluoroscopic images. Note that the use of a distance map-based 2-D/3-D registration algorithm facilitates robust tracking even if the 3-D model does not exactly match. As long as the 3-D model is registered to its associated 2-D projections in successive frames consistently, an accurate motion estimate can be obtained. In our experiment involving a moving-heart phantom, we found a mean 2-D tracking error of $1.4 \mathrm{~mm} \pm 0.6 \mathrm{~mm}$ with an average model error of $1.0 \mathrm{~mm}$. When evaluating clinical EP fluoroscopic sequences, we determined an average 2-D tracking error of $1.0 \mathrm{~mm}$ in the presence of an average model error of $0.4 \mathrm{~mm}$. The real tracking accuracy was therefore smaller than $1 \mathrm{~mm}$. The results presented show a slightly higher error for data acquired with the moving-heart phantom. We contribute this result to the fact that our method had been optimized for clinical data sets involving circumferential mapping catheters differrent from the one available for our experiments. Although unfortunate at first sight, this situation actually provided us with an opportunity to show that our method is robust by running it as is on the experimental data as well, i.e., without re-optimizing the algorithm parameters. The proposed method offers several advantages. First, it is workflow-friendly and does not require any fiducial markers or additional contrast agent. Second, 3-D motion is estimated directly at the site of ablation. There is no ambiguity coming from the inference of the real motion from surrogate motion estimates. Third, motion estimation and compensation is performed in one step. Therefore we do not need a motion model as part of the estimation algorithm. Fourth, our method does not place any restrictions on the 3-D data set that can be used. In other words, the fluoro overlay could be rendered from 3-D data sets acquired using MRI, CT, or C-arm CT such as syngo DynaCT Cardiac (Siemens AG, Forchheim, Germany). Since the motion of 
the LA can be approximated by a rigid-body transform [25], it is possible to apply the motion estimate obtained by 3-D catheter tracking to the static fluoroscopic overlay. This way, we can obtain an animated version of our initial overlay that moves in sync with the real anatomy. Figure 10 represents the conventional overlay technique without motion compensation, while Figure 11 shows an animated fluoroscopic overlay with motion compensation. With motion compensation, dynamic overlay and circumferential mapping catheter, fixed at the PV ostium, stay aligned. In addition, the contrast enhanced upper pulmonary vein, shown on the left side of the fluoroscopy image in Figure 11, matches well to the corresponding vein of the volumetric data. As far as accuracy is concerned, our 3-D motion error analysis yielded an average 3-D tracking error of $0.8 \mathrm{~mm}$ over 13 clinical data sets. Our 3-D tracking error is slightly lower than the 2-D tracking errors. This again demonstrates that accurate 3-D tracking can be achieved by simultaneously tracking within two 2-D imaging planes. This method is superior to existing methods that provide an accuracy of $2.0 \mathrm{~mm}[26,27]$, and it appears acceptable in clinical practice as our error is below $2 \mathrm{~mm}$ [28]. The current implementation of this algorithm achieves a frame rate of 3 frames-per-second using a single threaded CPU implementation. At clinical sites where this frame rate is used for EP procedures to keep X-ray dose low, real-time catheter tracking can be achieved already. In other cases, either a faster implementation is needed, or better hardware is required. These results demonstrate that model-based motion-compensation by $2-\mathrm{D} / 3-\mathrm{D}$ registration is both feasible and accurate.

\section{Acknowledgements}

The authors would like to thank Dr. med. Johannes Rieber for the gold-standard segmentation of the catheter, Andreas Wimmer for a tool to manually outline catheters easily, and Teri Moore for collecting the clinical data.

\section{References}

[1] Cappato, et al., "Worldwide Survey on the Methods, Efficacy, and Safety of Catheter Ablation for Human Atrial Fibrillation," Circulation 111, 1100-1105 (2005).

[2] Wazni, et al., "Radiofrequency ablation vs antiarrhythmic drugs as first-line treatment of symptomatic atrial fibrillation: a randomized trial," JAMA 293, 2634-2640 (2005).

[3] Zagorchev, et al., "Rapid fusion of 2D x-ray fluoroscopy with 3D multislice CT for imageguided electrophysiology procedures," in Proceedings of SPIE: Medical Imaging 2007: Visualization and Image-Guided Procedures, K. R. Cleary and M. I. Miga, Eds., 6509, pp. 65092B (2007).

[4] Sra, et al., "Computed Tomography-Fluoroscopy Image Integration-Guided Catheter Ablation of Atrial Fibrillation," J Cardiovasc Electrophysiol 18, 409-414 (2007).

[5] Strobel, et al., "Imaging with Flat-Detector C-Arm Systems," in Multislice CT (Medical Radiology / Diagnostic Imaging), M. F. Reiser, C. R. Becker, K. Nikolaou, and G. Glazer, Eds., ch. 3, 33-51, Springer Berlin / Heidelberg, third ed. (2009).

[6] Pruemmer, et al., "Cardiac C-arm CT: a unified framework for motion estimation and dynamic CT," IEEE Trans Med Imaging 28, 1836-1849 (2009).

[7] Lauritsch, et al., "Towards cardiac C-arm computed tomography," IEEE Trans Med Imaging 28, 922-34 (2006).

[8] Brost, et al., "3-D Respiratory Motion Compensation during EP Procedures by ImageBased 3-D Lasso Catheter Model Generation and Tracking," in MICCAI (1), G.-Z. Yang, D. J. Hawkes, D. Rueckert, J. A. Noble, and C. J. Taylor, Eds., Lecture Notes in Computer Science 5761, 394-401, Springer (2009).

[9] Timinger, et al., "Motion Compensated Coronary Interventional Navigation by Means of Diaphram Tracking and Elastic Motion Models," Phys. Med. Biol. 50, 491-503 (2005). 
[10] Ross, et al., "Motion Correction For Augmented Fluoroscopy - Application To Liver Embolization," in Proc IEEE Int Symp Biomed Imaging, ISBI, 1553-1556 (2008).

[11] Atasoy, et al., "Real-time Respiratory Motion Tracking: Roadmap Correction for Hepatic Artery Catheterizations," in Proceedings of SPIE Medical Imaging, 6918, 691815, (San Diego, CA, USA) (2008).

[12] Borgerta, et al., "Respiratory motion compensation with tracked internal and external sensors during CT guided procedures," International Congress Series 1281, 577-582 (2005).

[13] McLeish, et al., "A study of the motion and deformation of the heart due to respiration," IEEE Trans Med Imaging 21, 1142-1150 (2002).

[14] Shechter, et al., "Displacement and velocity of the coronary arteries: cardiac and respiratory motion," IEEE Trans Med Imaging 25, 369-375 (2006).

[15] Liao, et al., "Location Constraint Based 2D-3D Registration of Fluoroscopic Images of CT Volumes for Image-Guided EP Procedures," in Proceedings of SPIE Medical Imaging, 6918, 69182T-1-69182T-8 (2008).

[16] Halir, and Flusser, "Numerically Stable Direct Least Squares Fitting Of Ellipses," in In Proceedings of the 6th Conference in Central Europe on Computer Graphics and Visualization, 253-257, (Plzen) (1998).

[17] Fitzgibbon, et al., "Direct least square fitting of ellipses," IEEE Trans Pattern Anal Mach Intell 21, 476-480 (1999).

[18] Fitzgibbon and Fisher, "A Buyer's Guide to Conic Fitting," in BMVC '95: Proceedings of the 6th British conference on Machine Vision, 2, 513-522, BMVA Press, (Surrey, UK) (1995).

[19] L. Quan, "Conic Reconstruction and Correspondence From Two Views," IEEE Trans Pattern Anal Mach Intell 18, 151-160 (1996).

[20] Sato, et al., "3D Multi-Scale Line Filter for Segmentation and Visualization of Curvilinear Structures in Medical Images," Med Image Anal 2, 143-168 (1998).

[21] N. Otsu, "A Threshold Selection Method from Gray-Level Histograms," IEEE Trans Syst Man Cybern 9, 62-66 (1979).

[22] Breu, et al., "Linear time Euclidean distance transform algorithms," IEEE Trans Pattern Anal Mach Intell 17, 529-533 (1995).

[23] Brost, et al., "Accuracy of x-ray image-based 3D localization from two C-arm views: a comparison between an ideal system and a real device," in Medical Imaging 2009: Visualization, Image-Guided Procedures, and Modeling, M. I. Miga and K. H. Wong, Eds., 7261, 72611Z, SPIE, (Lake Buena Vista, FL, USA) (2009).

[24] Brost, et al., "Geometric Accuracy of 3-D X-Ray Image-Based Localization from Two CArm Views," in Workshop on Geometric Accuracy In Image Guided Interventions - Medical Image Computing and Computer Assisted Interventions 2009, L. Joskowicz, P. Abolmaesumi, and M. Fitzpatrick, Eds., 12-19, MICCAI, (London UK) (2009).

[25] Ector, et al., "Changes in Left Atrial Anatomy Due to Respiration: Impact on ThreeDimensional Image Integration During Atrial Fibrillation Ablation," J Cardiovasc Electrophysiol 19, 828-834 (2008).

[26] Baert, et al., "Guide Wire Tracking During Endovascular Interventions," IEEE Trans Med Imaging 22, 965-972 (2003).

[27] Elgort, et al., "Real-Time Catheter Tracking and Adaptive Imaging," J Magn Reson Imaging 18, 621-626 (2003).

[28] Esteghamatian, et al., "Real-time 2D-3D MR cardiac image registration during respiration using extended Kalman filter predictors," in 9th International Conference on Signal Processing (ICSP 2008), 1325-1328, IEEE, (Bejing, China) (2008). 